\title{
Berry phase and quantum structure
}

\author{
Holger Lyre \\ Philosophy Department \\ University of Magdeburg \\ Email: lyre@ovgu.de
}

August 2014

\begin{abstract}
The paper aims to spell out the relevance of the Berry phase in view of the question what the minimal mathematical structure is that accounts for all observable quantum phenomena. The question is both of conceptual and of ontological interest. While common wisdom tells us that the quantum structure is represented by the structure of the projective Hilbert space, the appropriate structure rich enough to account for the Berry phase is the $\mathrm{U}(1)$ bundle over that projective space. The Berry phase is ultimately rooted in the curvature of this quantum bundle, it cannot be traced back to the Hamiltonian dynamics alone. This motivates the ontological claim in the final part of the paper that, if one strives for a realistic understanding of quantum theory including the Berry phase, one should adopt a form of ontic structural realism.
\end{abstract}

\section{Introduction}

What is the structure of quantum theory? More precisely: what is the minimal structure that is necessary and sufficient to account for all quantum phenomena? Conventional wisdom tells us that it is the structure of a non-commutative algebra of observables represented by self-adjoint linear operators on a Hilbert space. Since, moreover, the representative of a state is given by a ray in Hilbert space, the state space even reduces to the projective Hilbert space. In this paper I argue that this answer is insufficient in so far as it misses an important element of structure known as the Berry phase. The Berry phase has considerable observable impact within a wide range of quantum phenomena. To understand its true nature one must scrutinize more rigorously the state space structure of quantum theory. It turns out that the appropriate structure to cover the Berry phase is a U(1) fiber bundle over the projective Hilbert space.

Albeit of central importance in quantum physics, philosophers of physics have largely neglected the Berry phase or, more generally, geometric and topological phases as a special topic. One 
notable exception is Batterman (2003) 1 But while Batterman thinks that the general lesson of this topic is rather epistemological, the focus of the present paper is on ontology. I will argue that, since the Berry phase is ultimately rooted in the state space structure of quantum theory itself, it is precisely the above-mentioned bundle structure that must be considered as real. This provides a direct link to structural realism as a doctrine that best captures the ontological commitments of the physics of Berry's geometric phase.

Basically, the paper consists of two chief claims: The first is that in order to account for all observable phenomena in connection with a given quantum system the projective Hilbert space structure is insufficient. It is the $\mathrm{U}(1)$ fiber bundle over the projective Hilbert space that is sufficient, I call it the quantum bundle. The second claim stresses the ontological commitment of the first claim, namely that the quantum bundle should be considered a real physical structure of nature. The main reason for this is that, as our analysis aims to show, the Berry phase cannot be traced back to the underlying Hamiltonian dynamics alone. It can neither be traced back to the existence of forces, nor to particular causal mechanisms, nor to the geometry or topology of spacetime. As a genuine quantum holonomy, the Berry phase is ultimately rooted in the quantum bundle structure itself. It is an outspring of the curvature of the quantum bundle, which leads to the ontological conclusion that Berry's phase supports ontic structural realism about the quantum bundle structure.

The paper is organized as follows: Section 2 starts with a portrayal of Berry's original derivation of the geometric phase. It was a major discovery, but it still masks the deeper nature of the phenomenon which only comes to the fore if the underlying fiber bundle structure is analyzed, as done in section 3. Berry's phase turns out as a holonomy of the quantum bundle. This will further be discussed by distinguishing various types of holonomies in section 4 with the result that Berry's phase is the sole instance of a geometric quantum phase. Section 5 introduces to what I dub "Batterman's challenge": the quest for an explanation of the ubiquity of holonomy phenomena in physics and their overall geometric understanding. Pace Batterman I will argue that a mere instrumental understanding of the physics of such phenomena is dissatisfying and that we should strive for an ontological picture. I conclude that, in the absence of a sufficient dynamical-cum-spacetime picture, Berry's geometric quantum phase gives us reason to consider the quantum bundle structure as an ontologically robust feature of the world. I end in section 6 with concluding remarks about a structural realist perspective regarding this structure.

\section{Berry's geometric quantum phase}

Consider a quantum system that undergoes a cyclic evolution such that final and initial states are identical. In general, the system will acquire a 'memory' of its evolution in terms of the so-called geometric phase of the wave-function. This leads to an observable phase shift. The idea was anticipated by Pancharatnam (1956), but was fully noted by Michael Berry only (Berry, 1984). It is therefore widely known as the Berry phase (informal introductions to the geometric phase can be found in Berry 1990 and Anandan 1992).

For a derivation of the geometric phase along the lines of Berry (1984) 2 consider a system with a Hamiltonian $\hat{H}_{R}$ that depends on a set of parameters $\vec{R}=\left(R_{1}, R_{2}, R_{3} \ldots\right)$. Assume that $\psi_{n, R}(t)$

\footnotetext{
${ }^{1}$ Another exception is Brown (1999), see also Siöqvist et al. (1997).

${ }^{2}$ For textbook treatments see Böhm et al. (2003) and Chruscinski and Jamiolkowski (2004).
} 
is a basis of eigenstates of $\hat{H}_{R}$ with non-degenerate eigenvalues $E_{n}$ obeying $\hat{H}_{R}\left|\psi_{n, R}\right\rangle=E_{n}\left|\psi_{n, R}\right\rangle$. We are interested in an adiabatic evolution along a circuit $C$ with period $T$ such that $\vec{R}(0)=$ $\vec{R}(T)$ holds. If the system is initially in state $\psi_{n, R}(0)$, the adiabadicity requirement guarantees that the system will not jump into another eigenstate but that later states $e^{i \phi_{n}(t)} \psi_{n, R}(t)$ will differ by a phase $\phi$ only. Berry discovered that $\phi$ will generally consist of two parts $\phi_{n}(t)=\delta_{n}(t)+\gamma_{n}(t)$. This can be seen by plugging $\tilde{\psi}_{n, R}(t)=e^{i \phi_{n}(t)} \psi_{n, R}(t)$ into the time-dependent Schrödinger equation $i \hbar \frac{d}{d t} \tilde{\psi}(t)=\hat{H}_{R} \tilde{\psi}(t)$ and projecting onto $\tilde{\psi}_{n, R}(t)$ :

$$
\frac{d}{d t} \phi_{n}(t)=i\left\langle\psi_{n, \vec{R}} \mid \vec{\nabla}_{\vec{R}} \psi_{n, \vec{R}}\right\rangle \frac{d}{d t} \vec{R}(t)-\frac{1}{\hbar} E_{n}
$$

Integration over $t$ yields the total phase consisting of the integral $\delta_{n}(t)=-\frac{1}{\hbar} \int E_{n}(t) d t$, called the dynamical phase representing the time evolution, and the "geometrical" phase $\gamma_{n}(t)$, which after a closed evolution yields

$$
\gamma_{n}(C)=i \oint_{C}\left\langle\phi_{n, \vec{R}} \mid \vec{\nabla}_{\vec{R}} \phi_{n, \vec{R}}\right\rangle d \vec{R}
$$

The derivation of Berry's geometrical phase within a pure quantum framework already indicates that we are dealing with a genuine quantum effect. Moreover, as Berry already noted, $\gamma_{n}$, is non-integrable, it cannot be written as a function of $\vec{R}$, but seems to depend on the particular path in $\vec{R}$-space - which is, curiously, an abstract mathematical parameter space (we will return to this point later).

Geometric quantum phases play a role in physics in a startling variety of phenomena ranging from optical physics, interference effects, crystal physics, nuclear magnetic resonance and others (Shapere and Wilczek (1989) give a comprehensive overview by providing a collection of relevant papers). The general setting of the observation of such phase is by interference of a cycled system with another uncycled system. A standard example is for instance a spin- $\frac{1}{2}$-particle in an adiabatically rotating magnetic field such that the spin aligns with the field. After a loop in spinor space the particle wave-function has acquired a complex phase. Another well-known example is Pancharatnam's already mentioned discovery of the phase shift of polarized light that is taken through a cycle of polarization states (Pancharatnam, 1956). It is, in retrospect, astonishing that such a broad class of phenomena was discovered as late as in the 1980's only, more than half a century since quantum theory had been fully developed.

\section{The fiber bundle structure of the quantum phase}

Soon after Berry's seminal work the geometric quantum phase has been recognized in its full generality. It is, as Simon (1983) has first pointed out (cf. also Page 1987), appropriately captured in the framework of fiber bundles and may, as Aharonov and Anandan (1987) were able to show, be generalized to non-adiabatic cases as well. From these two insights it turns out that the crucial structure from which the Berry phase originates is given by a $U(1)$-fiber bundle over the projective Hilbert space $\mathcal{H} P$. Call this the quantum bundle:

$$
U(1) \rightarrow \mathcal{H} \rightarrow \mathcal{H} P \quad \text { or } \quad \mathbb{P}(\mathcal{H} P, U(1)) .
$$

The quantum bundle defines a projection $\pi: \mathcal{H} \rightarrow \mathcal{H} P$ from the total bundle space, the Hilbert space $\mathcal{H}$, to the base space $\mathcal{H} P$. 
In the following subsection, a few remarks about projective spaces shall first be made. Next, the derivation of the Berry phase from the quantum bundle structure will be shown. It turns out that the Berry phase is in fact a holonomy that does not depend on the trajectory in parameter space but in the projective Hilbert space. This observation has crucial repercussions for our line of argument.

\subsection{Projective spaces}

In the case of $\mathbb{R}^{3}$ the corresponding projective space $\mathbb{R} P^{2}$ is the set of all lines in $\mathbb{R}^{3}$ passing through the origin. Note that every projection line meets a sphere centered in the origin exactly twice at antipodal points. Hence, $\mathbb{R} P^{2}$ is the set of points on the unit sphere $\mathbb{S}^{2}$ modulo an identification of antipodal points. Alternatively, it is the set of equivalence classes of points $(x, y, z) \in \mathbb{R}^{3} \backslash\{(0,0,0)\}$, where $(x, y, z)=(\lambda x, \lambda y, \lambda z)$ for a non-zero $\lambda \in \mathbb{R}$. It follows that $\mathbb{R} P^{2}$ is diffeomorphic to $\mathbb{S}^{2} / \mathbb{Z}_{2}$, where $\mathbb{Z}_{2}$ is the cyclic group of order 2 . More generally we get

$$
\mathbb{R} P^{n} \simeq \mathbb{S}^{n} / \mathbb{Z}_{2}
$$

The connection between projective spaces and quantum theory rests on the long known fact that the representative of a quantum state is not a vector but a ray in Hilbert space, i.e. a one-dimensional subspace or equivalence class $\psi \sim \lambda \psi$ with $\psi \in \mathcal{H}$ and $\lambda \in \mathbb{C}$. The principal argument for this is that the transformation $\psi \rightarrow \lambda \psi$ leaves the expectation values $\langle\hat{O}\rangle=\frac{\langle\psi|\hat{O}| \psi\rangle}{\langle\psi \mid \psi\rangle}$ of all observables $\hat{O}$ invariant. Hence, the projective Hilbert space $\mathcal{H} P$ as the set of rays is considered to be the state space of quantum theory. But this also leaves us with a puzzle. For even if we restrict ourselves to normalized state vectors by the requirement $\langle\psi \mid \psi\rangle=1$, as we standardly do, two states that differ by a phase $\psi^{\prime}=e^{i \varphi} \psi$ are equivalent. The phase difference drops off and phases seem to play no role in quantum theory any longer - at least if we follow the above line of argument. But not only does this render the original theory puzzlingly redundant, it also leaves no room to account for the Berry phase. The answer to this puzzle is that neither $\mathcal{H}$ nor $\mathcal{H} P$ gives us the necessary and sufficient structure to account for all observable phenomena. We must rather consider the bundle structure (3), a structure that sits, so to speak, right between $\mathcal{H}$ and $\mathcal{H} P$ (in the sense that, considered as sets, we have $\mathcal{H} P \subset \mathbb{P}(\mathcal{H} P, U(1)) \subset \mathcal{H}$ ). It is precisely this structure that accounts for the Berry phase.

\subsection{The quantum bundle $\mathbb{P}(\mathcal{H} P, U(1))$, its connection and holonomy}

Consider a finite $(n+1)$-dimensional quantum system with a Hilbert space $\mathcal{H}=\mathbb{C}^{n+1}$. Standard normalization of the states defines the sphere $\mathbb{S}^{2 n+1}$. The corresponding projective space is $\mathbb{C} P^{n} \simeq \mathbb{S}^{2 n+1} / \mathbb{S}^{1}$. The quantum theory of an $(n+1)$-dimensional system is then represented by the bundle

$$
U(1) \rightarrow \mathbb{S}^{2 n+1} \rightarrow \mathbb{C} P^{n} \simeq \mathbb{S}^{2 n+1} / \mathbb{S}^{1}
$$

The generalization of this result leads to the bundle structure (3) already introduced in the beginning of this section.

It will be sufficient to sketch the derivation here, the reader is referred to Böhm et al. (1991) and (2003) for details. A bundle connection serves to define the notion of parallel transport in a bundle and thus serves to identify neighboring fibers. Given the tangent space $T \mathbb{E}$ of a 
bundle $\mathbb{E}$ the connection is likewise a rule to define the horizontal subspace in the decomposition $T_{u} \mathbb{E}=V_{u} \mathbb{E}+H_{u} \mathbb{E}$ at any point $u \in \mathbb{E}$. Consider now tangent vectors $\dot{\varphi} \in T \mathbb{E}$ to the curve $\varphi \in \mathbb{E}$ which can be decomposed via the scalar product into

$$
|\dot{\varphi}\rangle=\left|v_{\varphi}\right\rangle+\left|h_{\varphi}\right\rangle=\langle\varphi \mid \dot{\varphi}\rangle|\varphi\rangle+\left|h_{\varphi}\right\rangle
$$

Note that two Hilbert space vectors that differ by a phase belong to the same fiber and point into the vertical fiber direction, hence the horizontal component satisfies $\left\langle\varphi \mid h_{\varphi}\right\rangle=0$. For the curve $\varphi$ to be a horizontal lift, the vertical part of (6) must vanish

$$
\langle\varphi \mid \dot{\varphi}\rangle=0 \text {. }
$$

A bundle holonomy is given by the horizontal lift of a closed curve $|\phi\rangle$ in base space with $|\phi(0)\rangle=|\phi(T)\rangle$ and $|\varphi\rangle=e^{i g(t)}|\phi\rangle$. Inserting this into (7) and integrating over $t$ yields

$$
\gamma=g(T)-g(0)=i \oint_{0}^{T}\langle\phi \mid \dot{\phi}\rangle d t
$$

We have, in full generality and without any recourse to an approximation (adiabatic or otherwise), re-discovered the quantum phase as the holonomy of the connection of the quantum bundle (3). This connection, known as the Berry connection, is simply the scalar product in Hilbert space.

In general, the phase depends of course on the choice of the connection. It is therefore important to note that Böhm et al. (1992) were able to show that the Berry connection is unique if we require invariance under the group of unitary transformations. Because of its direct connection to the conservation of probability, the unitary group can indeed be considered the canonical transformation group in quantum physics. The splitting between the dynamical and the geometrical phase is in this sense non-arbitrary, $\delta$ and $\gamma$ have independent physical meaning.

Important for the purposes of this paper is now the observation that unlike the derivation in section 2 the derivation of the generalized Berry-Aharonov-Anandan phase does not depend on the structure of the parameter space but on the projective Hilbert space. As Aharonov and Anandan (1987, p. 1594) put it: "we regard $\gamma$ as a geometric phase associated with a closed curve in the projective Hilbert space and not the parameter space, even in the special case considered by Berry." Moreover, $\gamma$ is reparametrization invariant and therefore independent of the rate or speed at which the evolution has occurred. In fact, an infinite number of Hamiltonians will generate motions in $\mathcal{H}$ which project onto the same curve in the projective Hilbert space and which therefore reproduce the same quantum phase.

In section 4, various types of holonomies will be distinguished and the particular type of the Berry phase as a geometric quantum phase will be explored. First, however, a short remark regarding the logico-algebraic structure of quantum theory: The discovery of the Berry phase and its deeper geometric nature has, it seems, repercussions for quantum theoretical programs that focus on the projective Hilbert space structure $\mathcal{H} P$ alone. This is seldom explicitly stated, but as we have seen, the necessary and sufficient structure to account for the Berry phase isn't $\mathcal{H} P$ but rather the $U(1)$ bundle over $\mathcal{H} P$. The structure of $\mathcal{H} P$ is most prominently studied in quantum logic, where one combines projective geometry with lattice theory (more precisely, the set of closed subspaces of the projective Hilbert space determines a special case of a non-Boolean orthomodular lattice). But the foregoing analysis sheds doubts on the prospects of quantum 
logic (or other programs that focus on the projective structure only) to be sufficient to account for the the Berry phase and, hence, for all quantum phenomena. The point is that the Berry phase is not represented by a self-adjoint operator, but as a holonomy of $\mathbb{P}(\mathcal{H} P, U(1))$. It is the quantum bundle structure (3) that must be considered the appropriate quantum structure.

On the other hand, to say that the bundle structure (3) is fundamental in quantum theory seems to create a tension in view of the standard operations in $\mathcal{H}$ such as the superposition of vectors and the action of operators, since they are defined on $\mathcal{H}$ as a vector space (an algebraic rather than a geometric structure). The tension, however, vanishes by the natural requirement that all such operations preserve the projection $\pi: \mathcal{H} \rightarrow \mathcal{H P}$. The transition probability for two states (rays), for instance, is defined by the 'angle' in the complex projective space that is fiber preserving (cf. Boya and Sudarshan 1989).

\section{The ubiquity of holonomies}

How does Berry's holonomy relate to other holonomies in physics? The ubiquity of physical holonomy phenomena calls for careful conceptual distinctions. Geometrical and topological effects can already be found in classical physics. We may distinguish between geometric/topological holonomies on the one hand and classical/quantum holonomies on the other as well as the Aharonov-Bohm holonomy as a special case.

\subsection{Geometric versus topological holonomies}

A familiar example of a classical holonomy is the parallel transport of a vector over a sphere along a loop. Consider the case where a vector is tangent to the sphere at the North pole. The vector may now be parallel transported along a great circle down to the equator, then further transported along the equator (say, a quarter of the whole equator) and taken back to the North pole along another great circle. Compared to its initial state the vector will now point into a different direction: it will have acquired a holonomy of $\pi / 2$.

Now consider the case of a Möbius strip. Take again a vector, but this time perpendicular to the surface. Transport it along the Möbius strip and after one circuit its direction will be reversed (and it will return to its initial position after another circuit). We have again produced a holonomy. But the two cases are different. In the case of the sphere the particular route is important and the holonomy will in general vary by a variation of the loop (suppose we had transported the vector along half of the equator: we would have produced a holonomy of $\pi$ ). In the case of the Möbius strip only multiples of a full circuit count and will solely produce two discrete holonomy outcomes: reversed $(-1)$ or non-reversed $(+1)$. Indeed, the holonomy in the case of the sphere or, more generally, in the case of curved spaces can be used to measure the curvature of the underlying space. The parallel transport is non-integrable, i.e. it depends on the particular path and does not depend on initial and final points only 3 The holonomy in the case of curved spaces can be traced back to the curvature properties of the underlying space. We may say that it detects the geometric properties of that space and can properly be called a

\footnotetext{
${ }^{3}$ Strictly speaking this must be called an anholonomy, since it relates to the distinction between integrable and non-integrable, anholonomic constraints in classical mechanics, but it is common language use in differential geometry to call it a holonomy as well.
} 
geometric holonomy. By way of contrast, the holonomy of the Möbius strip detects a topological feature of the underlying space and is therefore a topological holonomy.

The difference can very well be expressed in the language of fiber bundles. Our first scenario defines a tangent bundle over the sphere, while the Möbius strip represents a line bundle over the circle. Both bundles are non-trivial. In the general case of a tangent bundle on a curved space the bundle could either be trivial or non-trivial depending on the topological nature of the base space. Generally, a fiber bundle with total space $\mathbb{E}$, base space $\mathbb{B}$ and fiber $\mathbb{F}$ is trivial if it allows for a global section (think of the global structure as a cross product $\mathbb{E}=\mathbb{B} \times \mathbb{F}$ ). Obviously, the bundle is non-trivial if the base space has a non-trivial topology as in the cases of sphere and circle. More crucial for our purposes is the question whether the bundle has a vanishing or non-vanishing curvature tensor. In the former case, the connection, the derivative of which gives the curvature, is flat, while in the latter case the bundle has a non-flat connection. The tangent bundle over the sphere is a bundle with a non-flat connection. The Möbius strip, however, has a flat connection. Its holonomies are due to the non-trivial topology of the base space.

\subsection{Classical versus quantum geometric holonomies}

After highlighting the distinction between geometric and topological holonomies, we should consider the distinction between classical and quantum cases. The cases of parallel vector transport on the sphere or through a Möbius strip are classical cases. Another classical example is the following: classical oscillators that undergo, under special circumstances, a cyclic evolution exhibit a holonomy that is known as the Hannay angle (Hannay, 1985). This has often be pointed out as a classical analogue of the geometric quantum phase. A special and probably best known application is Foucault's pendulum. Here, the change of direction of the plane of swing can be described as a case of parallel transport by modeling the precession by the projection of the angular velocity of the Earth onto its normal direction. Foucault's pendulum is therefore a geometric holonomy in the same sense as the parallel transport of a vector on a sphere. By way of contrast, Berry's phase is a quantum phase in the sense that it is picked up by a wave-function. So it restricts to phases of quantum systems. At the same time, and as already pointed out in section 3. it depends on the cyclic evolution in the projective Hilbert space. It is in this sense a geometric quantum phase or, as it were, a "projective-geometric" quantum phase because of the fact that infinitely many Hamiltonians will generate motions in $\mathcal{H}$ projecting onto the same curve in $\mathcal{H} P$ and producing the same phase.

\subsection{The Aharonov-Bohm effect}

The Aharonov-Bohm effect (Aharonov and Bohm, 1959) deserves special attention since it is often considered to be an instance of the Berry phase. The scenario is very well known: a split electron beam passes around a solenoid in which a magnetic field is confined. The region outside the solenoid is field-free, but nevertheless a shift in the interference pattern on a screen behind the solenoid can be observed upon alteration of the magnetic field. The phase shift can be calculated from the loop integral over the potential, which - due to Stokes' theorem-relates to the magnetic flux

$$
\Delta \chi=q \oint_{\mathcal{C}} \vec{A} d \vec{r}=q \int_{\mathcal{S}} \vec{B} d \vec{s}=q \Phi_{m a g}
$$


The AB effect has triggered a host of theoretical considerations and experimental work (a comprehensive overview can be found in Peshkin and Tonomura 1989).

What is the nature of the $\mathrm{AB}$ effect? A first observation is that the $\mathrm{AB}$ effect is not of genuine quantum nature, since there exist classical $\mathrm{AB}$ scenarios. As a simple classical example consider the geometry of a cone with flat curvature (i.e. a vanishing gravitational field) everywhere except at the apex (which may be smoothed). Parallel transport on a loop enclosing the apex leads to a holonomy. The example provides a proper AB setting within classical (field) physics. The possibility of $\mathrm{AB}$ scenarios in both the classical and the quantum regime suggests that there is another structural feature to which the effect can be traced back. Common to the magnetic and electric $\mathrm{AB}$ effects in $\mathrm{QM}$ and gravitational $\mathrm{AB}$ cases is the fact that both underlying interactions, electromagnetism and gravity, can be described within the framework of gauge theories. This seems to indicate that the effect is tied to the underlying gauge theoretic structure (which is closely related to the claim that the fundamental entities in gauge theories are holonomies; cf. Healey 2007, Lyre 2004). This also fits to the fact that the AB effect can be generalized to arbitrary SU(N) gauge groups (Wu and Yang, 1975).

Moreover, the $\mathrm{AB}$ effect does not depend on the particular shape of the path if only the region of a non-vanishing gauge field strength is enclosed. This is the crucial similarity between the original magnetic $\mathrm{AB}$ effect and its classical gravitational analogue - and it relates to the above mentioned case of the topological holonomy of the Möbius strip in the specific sense that in this case, too, the holonomy does neither depend on the particular path nor does the path pass a region with non-vanishing curvature. That is, in both cases the connection is flat. In the AB case, however, it isn't flat throughout. What is characteristic of the AB scenario is that a region of a non-vanishing gauge field strength is enclosed (the confined magnetic field or the smoothed apex). It has, however, nothing to do with a non-trivial topology of the underlying base space. In both the original $\mathrm{AB}$ setting and in the case of the cone the base space is topologically well behaved and trivial. In this sense the $\mathrm{AB}$ effect is not a topological effect in the rigorous sense that it relates to the non-trivial topology of the underlying base space. The upshot is that the $\mathrm{AB}$ effect fits neither into the category of a pure topological nor a pure geometric holonomy, it depends on the existence of a confined gauge field. It is thus no instance of the Berry phase.

\section{Batterman's challenge and minimal quantum structure}

We have seen from section 2 that the Berry phase plays an eminent role throughout quantum physics and for a large spectrum of phenomena. Albeit of great experimental and theoretical importance, philosophers of physics have largely overlooked Berry's quantum phase as a special topic. Given the experimental evidence this is indeed a serious neglect. One notable exception is Robert Batterman (2003). Batterman's paper draws attention to the ubiquity of holonomies by highlighting many important physical and mathematical facts. His discussion runs over many of the issues we already touched upon in the foregoing sections: ideas of parallel transport, Pancharatnam's polarization phase, Foucault's pendulum, the AB effect, fiber bundles, and so on. As Batterman emphasizes, his focus is on the explanatory value of such geometric phenomena rather than on ontology. He encounters a challenging puzzle: "It turns out that you can explain the physical changes that appear as a result of ... round trip excursions by appeal to certain purely geometrical features of the abstract space in which the excursion can be parameterized. This is, 
prima facie, odd. What sort of role can geometrical/topological features of some abstract space play in explaining and providing understanding of "real" physical phenomena?" (Batterman 2003, p. 527)

Indeed, that sounds odd. If real physical phenomena were to depend on the properties of some abstract, mathematical parameter space, then we seem to be left with an unfortunate alternative: either we come to the conclusion that our physical explanation of the phenomena is strictly speaking wrong, since it doesn't provide us with a full-blown explanation drawing on either causal mechanisms, forces, or, as we will discuss below, spacetime geometry, or we bite the bullet and come to terms with Platonism. Batterman - rather implicitly than explicitly - seems to endorse the first option. He acknowledges the ubiquity of geometric and topological phenomena in physics, and argues that the universality of such phenomena calls for unifying and wideranging explanations. The description in terms of holonomies provides us with such a universal explanation, but, as Battermain claims, without any need to take it metaphysically serious. After all, in all those types of explanations certain crucial idealizations and approximations are involved.

However, caution is demanded. Is it really true that idealizations alone account for the ubiquity of holonomies? Idealizations and approximations are Batterman's special topic (compare Batterman 2002, 2010), and they obviously play an eminent role in many places in physics and the sciences throughout. But certainly not all explanations in terms of holonomies are explanations which invoke idealizations or approximations. Holonomies are no mysteries. In many cases we are able to tell a causal-mechanistic story (including physical forces), other cases allow us to tell a story in terms of the underlying geometry of position space.

Consider again the case of parallel vector transport in curved space. Obviously, what is responsible for the occurrence of holonomies in such a case is the curvature of the underlying base space. Likewise the case of the Möbius strip. What brings about this type of holonomy is the non-trivial topology of the base space. We have thus two simple cases, cases of classical holonomies, for which a non-mysterious story can very easily be told.

By way of contrast, consider Foucault's pendulum. Here, the description in terms of parallel transport and holonomies is really just an idealized way to describe what's going on. What brings about the precession of the oscillation plane of Foucault's pendulum? From the perspective of an earth-bound reference frame, it can be seen as an effect of the Coriolis force. But since this force is a pseudo-force reflecting the rotation of the earth, it is the rotation of the earth itself that is ultimately responsible for Foucault's holonomy. This raises the suspicion that in all cases of classical holonomies the effect can either be traced back to the influence of forces or has to do with the geometrical or topological properties of position space itself as the underlying base space. No mysterious abstract spaces or idealized explanations must be invoked.

A rather peculiar case is the special relativistic case of Thomas precession 4 Consider a gyroscope travelling along a loop in flat Minkowski spacetime. After the loop the gyroscope's axis will not align with its original direction, it has acquired a holonomy. What brings about this effect? No forces are acting on the gyroscope, nor is position space curved. Standard treatments make reference to the fact that the space of velocities in special relativity is hyperbolic, and so the parallel transport of a vector changes its direction. Should we say that the gyroscope detects the geometry of velocity space, which then brings about the precession effect?

\footnotetext{
${ }^{4}$ I like to thank Harvey Brown and David Malament for independently pointing this out to me.
} 
One could put it this way, we should however recognize the fact that, unlike classical Euclidean spacetime, the geometry of Minkowski spacetime mandates that translations and momenta are non-trivially connected, and only together form the geometrical symmetry group of special relativity. Thomas precission can algebraically be understood as an outspring of the Lorentz group (more precisely, the non-associativity of velocities). So even if the effect cannot be traced back to the curvature of position space, it still reflects the geometrical symmetry of Minkowski spacetime. Again: classical holonomies can either be traced back to forces or to the geometrical structure of spacetime, or so it seems 5 The real threat comes with quantum holonomies.

What brings about the Berry phase? The effect is neither brought about by forces nor by the geometry of spacetime. Take the shift of the wave-function of a spin- $\frac{1}{2}$-particle in a rotating magnetic field. Of course, the rotation of the magnetic field is a force- or interaction-like process. But it alone cannot account for Berry's phase. Moreover, as we have already seen, an infinite number of Hamiltonians may produce the same phase. The Hamiltonian dynamics leading to the cyclic evolution in $\mathcal{H} P$ is at best a necessary, but not a sufficient condition for Berry's phase. What is crucial, however, is the fact that $\mathcal{H} P$ has a special geometry. So what brings about the effect? On the basis of the bundle-theoretic derivation in section 3 we are now in the position to give a precise answer to this question: It is the curvature of the quantum bundle that brings about Berry's phase. Any quantum evolution is constrained by the geometry of (31), the $U(1)$ bundle over the projective Hilbert space. Berry's quantum phase detects, as it were, the quantum bundle curvature.

This is remarkable, since this is the first instance of a physical holonomy in our discussion so far that draws on a piece of physical structure that exceeds dynamical-cum-spacetime structure. It also becomes clear that the structure of the quantum bundle is the minimal structure that is necessary and sufficient to account for all quantum phenomena. The structure of the quantum world cannot be accounted for by the structure of $\mathcal{H} P$, but only by the structure of a $U(1)$ bundle over $\mathcal{H} P$.

It is this extra piece of quantum structure that provides us with a third option regarding Batterman's challenge that goes beyond the alternative of either idealizations due to a missing dynamical-cum-spacetime picture or Platonism. The third option is that, since the Berry phase can be rediscovered as a holonomy of the Berry connection, the existence of that phase is directly rooted in the quantum bundle structure. Batterman's analysis misses this option since he only refers to Berry's original derivation, which traces the phase back to parameter space (as shown in section (2). If this were the end of the story, this would indeed be "odd". Berry's phase, however, doesn't live in parameter space, but in the quantum bundle. It detects the curvature of that bundle, any quantum loop evolution is constrained by it.

But why not consider the quantum bundle structure as an idealization already? The reason is that even an instrumentalist or operationalist about quantum theory must use this much structure at minimum to account for all observable quantum phenomena. He cannot just work with the structure of $\mathcal{H} P$, only the $U(1)$ bundle over $\mathcal{H} P$ will do.

\footnotetext{
${ }^{5}$ I leave this as a hypothesis. Nothing hinges on its truth for the purposes of this paper, which ultimately focuses on the quantum Berry phase).
} 


\section{Quantum structure and structural realism}

The aim of the paper was to spell out the relevance of the Berry phase in view of the question what the minimal mathematical structure is that accounts for all observable quantum phenomena. This aim has been reached by identifying the quantum bundle (3) as the minimally relevant structure. The paper could stop at this point, but in this final section I want to go one step beyond a merely conceptual analysis of the structure of quantum theory and draw possible ontological conclusions from it. Non-realists will surely not follow, but the scientific realist, who is willing to make an ontological commitment, cannot overlook the relevance of the quantum bundle as the minimal quantum structure. It is the purpose of this final section to spell out a moderate and at the same time straightforward realist commitment in connection with the above findings. My recommendation is to be a realist about the quantum bundle structure reflecting the quantum structure of the physical world.

Let me emphasize that it is not the purpose of this paper to present arguments in favor of structural realism per se 6 I rather want to argue that, if one aims for an ontological picture of quantum physics including the Berry phase, structural realism appears to be a promising, if not the most promising option. Philosophers of Batterman's stripe will certainly not be convinced. They will stick with the view that sometimes abstract mathematical structures can play an explanatory role in empirical science - no further comments necessary. However, in the absence of a missing dynamical-cum-spacetime picture, I find this highly dissatisfying (as elaborated on in the previous section) and therefore recommend to look for an ontologically robust interpretation. The opposite side might insist that it is as odd to consider the quantum bundle structure as real as to consider Berry's original parameter space as real. But note that there is a vast number of Berry phase scenarios with a vast number of particular parameter spaces. It would be inflationary (and crazy) to consider all of them as "real." Fortunately, there is only one quantum bundle to account for this vast number of observable effects. Its structure (basically encoded in the bundle connection) is, as we've seen, the minimal structure necessary and sufficient to account for all observable quantum phenomena. It is precisely this minimality feature that makes it a distinguished piece of structure and thus a candidate structure for the quantum structural realist. And there is nothing mysterious about the quantum bundle structure as compared to other candidate structures, mostly groups and algebras, that have already been identified by SR proponents by the same rationale.

Let me elaborate on this. Quite generally, the scientific realist who takes quantum theory serious will acknowledge that the world is of quantum nature - which is to say that it has a quantum structure. That structure is naturally encoded into the quantum theoretical state space: it tells us how physical states are related to each other. But as we have seen, the quantum physical state space $\mathcal{H} P$ not only encodes the state transitions in terms of its algebraic structure (as quantum logic would have it), but also constraints the temporal evolution of quantum states in a subtle geometrical way such that, in the case of loop evolutions, holonomies occur. It seems therefore natural to advocate a form of realism about the quantum bundle structure and, hence, to advocate a form of ontic structural realism (OSR).

To motivate our structural move in a more "relationalist" fashion consider the following: For the Berry phase to become manifest and observable we need to compare two systems. Perhaps

\footnotetext{
${ }^{6}$ I've done this elsewhere, e.g. Lyre 2004, 2012; see also French (2014) for a recent comprehensive anthology on structural realism.
} 
the peculiarity of the Berry phase lies in the fact that it exists only as a relation between two systems? A critic might indeed point out that the effect is manifest as a relative phase only and that there is nothing special about relative phases in quantum theory. It is true that relative quantum phases are in principe observable. Take a quantum beam splitter, e.g. a double slit, and consider a phase shifter in one of the beams, then surely the relative phase becomes observable as a shift in the interference fringe. But note again that this effect is perfectly understandable in terms of the (more or less) locally acting phase shifter. This, again, is not the case for the Berry phase. It is true that it becomes observable as a relative phase between two systems only, an evolved system and a reference system, but the change in the relative phase or, more generally, the change in the relation between the two systems is not brought about by any forces or the like. So, how does Nature do it? What ontological picture accounts for a world that shows effects like this?

At this point a structuralist move suggests itself. SR assumes (at least in its moderate version) that on the fundamental level there are otherwise non-individuated objects with relational properties only. Such relations are globally orchestrated by the (various) structure(s) of the world. Structural realists should thus be prepared that Nature behaves such that relational changes might occur without the existence or influence of locally acting forces. The Berry phase is thus an outspring of the quantum structure of the world. More precisely: In a quantum world it is to be expected that relational changes in terms of Berry's geometrical holonomy will occur, since this holonomy is built into the world's quantum structure. There is no quantum world without Berry phases. The relational changes spelled out by the Berry phase are not brought about by forces, they are built into Nature's bottom structure. From an ontological point of view, we may account best for this effect by assuming an ontological picture that does not construe the world to consist of a mosaic of local entities, but rather to consist of global structures.

One might compare the quantum bundle structure to the constraint that permutation symmetry imposes on the allowed physical states in a many particle state space. Here, too, the permutation symmetry structure brings about real physical effects, frequently spelled out in connection with Pauli's exclusion principle. An impressive instantiation of this is, for instance, the electron degeneracy pressure that leads to the stability of white dwarfs and neutron stars by preventing them from collapsing under their own weight. OSR proponents will therefore also take the permutation symmetry structure as a real ingredient of our physical quantum world.

Note that OSR isn't Platonism, in fact, it is quite the opposite of Platonism. Of course, fiber bundles, Hilbert spaces, and the like are mathematical entities in the first place. Ontic in restructural realism is, however, the view that the basic structures that occur in the mathematical representation of our best and mature physical theories correspond to existing structures in the world (as opposed to the Platonist ante rem-picture of structures). In the same sense should the quantum bundle structure be understood as a physical in re-structure of the world.

The most prominent example of in re-structures that has been invoked by structural realists so far is symmetry group structure (cf. Kantorovich 2003, Lyre 2004). Brian Roberts (2011, p. 50) has aptly called this position "Group Structural Realism (GSR)," and he defines it in the following way: "The existing entities described by quantum theory are organized into a hierarchy, in which a particular symmetry group occupies the top, most fundamental position." Roberts adds that "this statement of GSR should be taken as a minimal assumption of the view" allowing us "to leave the exact nature of a group structure's 'existence' to the individual metaphysician." 
In the same spirit, we may broaden the scope of GSR by considering more than just groups as physical structures. Indeed, fundamental physics tells us that other structures are manifested as well. Among the candidate structures that modern physics invites us to consider are, most prominently, the external spacetime symmetry structure, the internal gauge groups structure, and the quantum structure.

Structural realism is at the same time a moderate version of realism. A full-blown realism about the quantum bundle would be a bundle space substantivalism. But as in the debate about space-time ontology, where relationalists (anti-realists) and substantivalists (realists) about spacetime stand opposed, structural realism offers a third, intermediate route. This fits nicely to the observation about Thomas precession in the foregoing section, where it turned out that this effect is covered as long as one takes the Lorentzian structure of spacetime into account - which means, ontologically speaking, that one should consider it a real trace of nature. Minkowski spacetime is real insofar as it consists of a structure that constrains the geodesic behavior of particles as well as the direction of gyroscopes. Spacetime structuralism, unlike full-blown substantivalism, only takes this much spacetime structure as real. Along the same lines, it is only the structure of the quantum bundle that I advocate to consider as real. As its most crucial structural feature this structure entails the possibility of holonomies, which is just what the Berry phase is.

To conclude: Berry's quantum phase is neither brought about by the existence of forces nor by the geometrical or topological properties of spacetime, but rather by the geometrical structure of a U(1) fiber bundle over the projective Hilbert space. And for precisely the same reasons why (structural) realists are well-advised to consider the external space-time structure, the internal gauge group structure, and the permutation symmetry structure of quantum theory as existing, they are also advised to consider the quantum bundle structure as real.

\section{References}

Aharonov, Y. and J. Anandan (1987). Phase Change during a Cyclic Quantum Evolution. Physical Review Letters 58: 1593-1596.

Aharonov, Y. and D. Bohm (1959). Significance of Electromagnetic Potentials in the Quantum Theory. Physical Review 115(3): 485-491.

Anandan, J. (1992). The Geometric Phase. Nature 360: 307-313.

Batterman, R. (2002). The Devil in the Details: Asymptotic Reasoning in Explanation, Reduction, and Emergence. New York: Oxford University Press.

Batterman, R. (2003). Falling Cats, Parallel Parking, and Polarized Light. Studies in History and Philosophy of Modern Physics 34(4): 527-557.

Batterman, R. (2010). On the Explanatory Role of Mathematics in Empirical Science. The British Journal for the Philosophy of Science 61(1): 1-25.

Berry, M. (1984). Quantal Phase Factors Accompanying Adiabatic Changes. Proceedings of the Royal Society of London A 392: 45-57.

Berry, M. (1990). Anticipations of the Geometric Phase. Physics Today 12: 34-40. 
Böhm, A., L. J. Boya and B. Kendrick (1991). Derivation of the Geometrical Phase. Physical Review A 43(3): 1206-1210.

Böhm, A., L. J. Boya and B. Kendrick (1992). On the Uniqueness of the Berry Connection. Journal of Mathematical Physics 33(7): 2528-2532.

Böhm, A., Q. Niu, A. Mostafazadeh, H. Koizumi and J. Zwanziger (2003). The geometric phase in quantum systems. New York: Springer.

Boya, L. J. and E. C. G. Sudarshan (1989). Rays and Phases in Quantum Mechanics. Foundations of Physics Letters 4(3): 283-287.

Brown, H. R. (1999). Aspects of Objectivity in Quantum Mechanics. In: J. Butterfield and C. Pagonis, eds.: From Physics to Philosophy, Cambridge: Cambridge University Press.

Chruściński, D. and A. Jamiołkowski (2004). Geometric Phases in Classical and Quantum Mechanics. Boston: Birkhäuser.

French, S. (2014). The Structure of the World: Metaphysics and Representation. Oxford: Oxford University Press.

Hannay, J. H. (1985). Angle Variable Holonomy in Adiabatic Excursion of an Integrable Hamiltonian. Journal of Physics A 18: 221-230.

Healey, R. (2007). Gauging What's Real. New York: Oxford University Press.

Lyre, H. (2004). Holism and Structuralism in U(1) Gauge Theory. Studies in History and Philosophy of Modern Physics 35(4): 643-670.

Lyre, H. (2012). Structural Invariants, Structural Kinds, Structural Laws. In: Dieks, D. et al., eds.: Probabilities, Laws, and Structures, pp. 169-181. Springer, Dordrecht.

Kantorovich, A. (2003). The Priority of Internal Symmetries in Particle Physics. Studies in History and Philosophy of Modern Physics 34: 65175.

Page, D. N. (1987). Geometrical Description of Berry's Phase. Physical Review A 36: 3479-3481.

Pancharatnam, S. (1956). Generalized Theory of Interference, and Its Applications. Part I. Coherent Pencils. Proceedings of Indian Academy of Sciences A 44: 247-262.

Peshkin, M. A. and A. Tonomura (1989). The Aharonov-Bohm Effect. Lecture Notes in Physics 340. Berlin: Springer.

Roberts, B. W. (2011). Group Structural Realism. The British Journal for the Philosophy of Science 61(1): 47-69.

Shapere, A. and F. Wilczek, eds. (1989). Geometric Phases in Physics. Singapore: World Scientific.

Simon, B. (1983). Holonomy, the Quantum Adiabatic Theorem, and Berry's Phase. Physical Review Letters 51(24): 2167-2170.

Sjöqvist, E., H. R. Brown and H. Carlsen (1997). Galilean Noninvariance of Geometric Phase. Physics Letters A 229: 273-278.

Wu, T. T. and C. N. Yang (1975). Concept of Nonintegrable Phase Factors and Global Formulation of Gauge Fields. Physical Review D 12(12): 3845-3857. 